\title{
FRIICAT: A FIRST catalog of FR II radio galaxies ^
}

\author{
A. Capetti ${ }^{1}$, F. Massaro ${ }^{2}$, and R. D. Baldi ${ }^{3}$ \\ 1 INAF-Osservatorio Astrofisico di Torino, via Osservatorio 20, 10025 Pino Torinese, Italy \\ e-mail: capetti@oato.inaf.it \\ 2 Dipartimento di Fisica, Università degli Studi di Torino, via Pietro Giuria 1, 10125 Torino, Italy \\ 3 Department of Physics and Astronomy, University of Southampton, Highfield, SO17 1BJ, UK
}

Received 14 December 2016 / Accepted 30 January 2017

\begin{abstract}
Aims. We built a catalog of 122 FR II radio galaxies, called FRIICAT, selected from a published sample obtained by combining observations from the NVSS, FIRST, and SDSS surveys. The catalog includes sources with redshift $\leq 0.15$, an edge-brightened radio morphology, and those with at least one of the emission peaks located at radius $r$ larger than $30 \mathrm{kpc}$ from the center of the host. Methods. The radio luminosity at $1.4 \mathrm{GHz}$ of the FRIICAT sources covers the range $L_{1.4} \sim 10^{39.5}-10^{42.5} \mathrm{erg} \mathrm{s}^{-1}$. The FRIICAT catalog has $90 \%$ of low and 10\% of high excitation galaxies (LEGs and HEGs), respectively. The properties of these two classes are significantly different. The FRIICAT LEGs are mostly luminous $\left(-20 \gtrsim M_{r} \gtrsim-24\right)$, red early-type galaxies with black hole masses in the range $10^{8} \lesssim M_{\mathrm{BH}} \lesssim 10^{9} M_{\odot}$; they are essentially indistinguishable from the FR Is belonging to the FRICAT. The HEG FR IIs are associated with optically bluer and mid-IR redder hosts than the LEG FR IIs and to galaxies and black holes that are smaller, on average, by a factor $\sim 2$.

Results. FR IIs have a factor $\sim 3$ higher average radio luminosity than FR Is. Nonetheless, most ( $\sim 90 \%)$ of the selected FR IIs have a radio power that is lower, by as much as a factor of $\sim 100$, than the transition value between FR Is and FR IIs found in the $3 \mathrm{C}$ sample. The correspondence between the morphological classification of FR I and FR II and the separation in radio power disappears when including sources selected at low radio flux thresholds, which is in line with previous results. In conclusion, a radio source produced by a low power jet can be edge brightened or edge darkened, and the outcome is not related to differences in the optical properties of the host galaxy.
\end{abstract}

Key words. galaxies: active - galaxies: jets

\section{Introduction}

Fanaroff \& Riley (1974) introduced the first classification scheme for extragalactic radio sources with large-scale structures (i.e., greater than $\sim 15-20 \mathrm{kpc}$ in size) based on the ratio $R_{\mathrm{FR}}$ of the distance between the regions of highest surface brightness on opposite sides of the central host galaxy to the total extent of the source up to the lowest brightness contour in the radio images. Radio sources with $R_{\mathrm{FR}}<0.5$ were placed in Class I (i.e., the edge-darkened FR Is) and sources with $R_{\mathrm{FR}}>0.5$ in Class II (i.e., the edge-brightened FR IIs). This morphology-based classification scheme was found to be linked to their intrinsic power; Fanaroff and Riley found that all sources in their sample with luminosity at $178 \mathrm{MHz}$ smaller than $2 \times 10^{25} \mathrm{~W} \mathrm{~Hz}^{-1} \mathrm{sr}^{-1}$ (for a Hubble constant of $50 \mathrm{~km} \mathrm{~s}^{-1} \mathrm{Mpc}^{-1}$ ) were classified as FR I while the brighter sources all were FR II. The luminosity distinction between FR classes is fairly sharp at $178 \mathrm{MHz}$; their separation is even cleaner in an optical-radio luminosity plane, implying that the FR I/FR II dichotomy depends on both optical and radio luminosity (Ledlow \& Owen 1996).

The two Fanaroff-Riley classes do not instead correspond to a division from the point of view of the optical spectroscopic properties of their hosts. Laing et al. (1994) defined low and high excitation galaxies (LEGs and HEGs) based on the ratios of the diagnostic optical emission lines in a scheme similar

\footnotetext{
* Table 1 is only available at the CDS via anonymous ftp to cdsarc.u-strasbg. fr (130.79.128.5) or via http://cdsarc.u-strasbg.fr/viz-bin/qcat?J/A+A/601/A81
}

to that adopted to distinguish LINERs and Seyferts in radio quiet AGN (Kewley et al. 2006). While all FR Is for which a reliable classification can be obtained are LEGs, both LEGs and HEGs are found among the FR IIs (e.g., Buttiglione et al. 2010). Buttiglione et al. find that LEGs and HEGs also differ from other points of view. While LEGs do not show prominent broad lines, they are observed in $\sim 30 \%$ of HEGs; narrow emission lines are a factor $\sim 10$ brighter in HEGs than in LEGs at the same radio luminosity. Also, HEGs show bluer colors than LEGs (Smolčić 2009). Baldi \& Capetti (2008) identify compact knots in the UV images of 3C HEG radio galaxies, a morphological evidence of recent star formation extending over 5-20 kpc; conversely, LEGs hosts are usually red, passive galaxies. These results suggest that the radio galaxies belonging to the two spectroscopic classes correspond to different manifestation of the radio loud AGN phenomenon (Hardcastle et al. 2007; Buttiglione et al. 2010; Best \& Heckman 2012).

The recent multiwavelength large-area surveys are a unique tool to further explore the connection between the morphological and spectroscopic classes of radio galaxies, providing us with large samples of radio emitting AGN extending to lower luminosities than in previous studies.

In Capetti et al. (2017) we created a catalog of 219 edgedarkened FR I radio galaxies called FRICAT. We found that the FRICAT hosts are remarkably homogeneous, as they are all luminous red early-type galaxies (ETGs) with large black hole masses and spectroscopically classified as LEGs. All these properties are shared by the hosts of more powerful FR Is in the 
3C sample. They do not show significant differences from the point of view of their colors with respect to the general population of massive ETGs. The presence of an active nucleus (and its level of activity) does not appear to affect the hosts of FR Is.

We now extend this study to the population of edgebrightened FR II radio galaxies with the main aim of comparing the properties of FR Is and FR IIs by also considering their spectroscopic classification.

This paper is organized as follows. In Sect. 2 we present the selection criteria of the sample of FR IIs. The radio and optical properties of the selected sources are presented in Sect. 3. Section 4 is devoted to results and conclusions.

Throughout the paper we adopt the same cosmology parameters used in Capetti et al. (2017), i.e., $H_{0}=67.8 \mathrm{~km} \mathrm{~s}^{-1} \mathrm{Mpc}^{-1}$, $\Omega_{\mathrm{M}}=0.308$, and $\Omega_{\Lambda}=0.692$ (Planck Collaboration XIII 2016).

For our numerical results, we use c.g.s. units unless stated otherwise. Spectral indices $\alpha$ are defined in the usual convention on the flux density, $S_{v} \propto v^{-\alpha}$. The SDSS magnitudes are in the $\mathrm{AB}$ system and are corrected for the Galactic extinction; WISE magnitudes are instead in the Vega system and are not corrected for extinction since, as shown by, for example, D'Abrusco et al. (2014), such correction affects mostly the magnitude at $3.4 \mu \mathrm{m}$ of sources lying at low Galactic latitudes (and by less than $23 \%$ ).

\section{Sample selection}

We searched for FR II radio galaxies in the sample of 18286 radio sources built by Best \& Heckman (2012, hereafter the BH12 sample) by limiting our search to the subsample of objects in which, according to these authors, the radio emission is produced by an active nucleus. They cross-matched the optical spectroscopic catalogs produced by the group from the Max Planck Institute for Astrophysics and Johns Hopkins University (Brinchmann et al. 2004; Tremonti et al. 2004) based on data from the data release 7 of the Sloan Digital Sky Survey (DR7/SDSS; Abazajian et al. 2009) ${ }^{1}$. with the National Radio Astronomy Observatory Very Large Array Sky Survey (NVSS; Condon et al. 1998) and the Faint Images of the Radio Sky at Twenty centimeters survey (FIRST; Becker et al. 1995; Helfand et al. 2015) adopting a radio flux density limit of $5 \mathrm{mJy}$ in the NVSS. We focused on the sources with redshift $z<0.15$.

We adopted a purely morphological classification based on the radio structure shown by the FIRST images. We visually inspected the FIRST images of each source and preserved those with an edge brightened morphology in which at least one of the emission peaks lies at a distance of at least $30 \mathrm{kpc}$ from the center of the optical host. The $30 \mathrm{kpc}$ radius corresponds to $11^{\prime \prime} .4$ for the farthest objects; the $z<0.15$ redshift limit ensures that all the selected sources are well resolved with the $5^{\prime \prime}$ resolution of the FIRST images. The three authors performed this analysis independently and we included only the sources for which a FR II classification is proposed by at least two of us. We allowed for the presence of diffuse emission leading to X-, Z-, or C-shaped morphologies, but not extending at larger distances with respect to the emission peaks, thus excluding wide angle tail sources (Owen \& Rudnick 1976). Most of these sources are double, i.e., they do not show nuclear radio emission; the lack of this precise position reference requires a further check of the original optical identifications. We discarded three objects in which the identification of the host is not secure.

Available at http://www .mpa-garching.mpg.de/SDSS/
The resulting sample, to which we refer as FRIICAT, is formed by 122 FR IIs whose FIRST images are presented in the Appendix. Their main properties are presented in Table 1, where we report the SDSS name, redshift, and NVSS 1.4 GHz flux density (from BH12). The [O III] line flux, the $r$-band SDSS AB magnitude, $m_{r}$, the Dn(4000) index (see Sect. 3 for its definition), and the stellar velocity dispersion $\sigma_{*}$ are instead from the MPA-JHU DR7 release of spectrum measurements. The concentration index $C_{r}$ was obtained for each source directly from the SDSS database. For sake of clarity, uncertainties are not shown in the table; we estimated a median uncertainty of 0.09 on $C_{r}$, of 0.03 on Dn(4000), of 0.005 magnitudes on $m_{r}$, and of $10 \mathrm{~km} \mathrm{~s}^{-1}$ on $\sigma_{*}$. We also list the resulting radio and line luminosity and the black hole masses estimated from the stellar velocity dispersion and the relation $\sigma_{*}-M_{\mathrm{BH}}$ of Tremaine et al. (2002). The uncertainty in the $M_{\mathrm{BH}}$ value is dominated by the spread of the relation used (rather than by the errors in the measurements of $\sigma_{*}$ ) resulting in an uncertainty of a factor $\sim 2$. Finally, we give the classification (from BH12) into LEGs and HEGs based on the optical emission line ratios in their SDSS spectra.

\section{FRIICAT hosts and radio properties}

\subsection{Hosts properties}

The majority (107) of the selected FR IIs are classified as LEG, but there are also 14 HEG and just one source that cannot be classified spectroscopically because of the lack of emission lines, namely $\mathrm{J} 1446+2142$.

The distribution of absolute magnitude of the FRIICAT hosts covers the range $-20 \gtrsim M_{r} \gtrsim-24$ (see Fig. 1, left panel). The distribution of black hole masses (Fig. 1, right panel) is rather broad. Most sources have $8.0 \lesssim \log M_{\mathrm{BH}} \lesssim 9.0 M_{\odot}$, but a tail toward smaller values, down to $M_{\mathrm{BH}} \sim 10^{6.5} M_{\odot}$, that includes $\sim 15 \%$ (13 LEGs and 4 HEGs) of the sample.

The FR II HEGs are less luminous, overall, and harbor less massive black holes with respect to the FR II LEGs; the medians of their distributions are $\left\langle M_{r}(\mathrm{HEG})\right\rangle=-21.97 \pm 0.17$, $\left\langle M_{r}(\mathrm{LEG})\right\rangle=-22.62 \pm 0.06,\left\langle\log M_{\mathrm{BH}}(\mathrm{HEG})\right\rangle=8.21 \pm 0.11$, and $\left\langle\log M_{\mathrm{BH}}(\mathrm{LEG})\right\rangle=8.46 \pm 0.04$, respectively. The comparison between the FRIICAT LEG sources and the FRICAT hosts $\left(\left\langle M_{r}(\mathrm{FR} \mathrm{I})\right\rangle=-22.69 \pm 0.03\right.$ and $\left.\left\langle\log M_{\mathrm{BH}}(\mathrm{FR} \mathrm{I})\right\rangle=8.55 \pm 0.02\right)$ indicates that only marginal differences (and not statistically significant) are present between the medians of these distributions.

The Dn(4000) spectroscopic index, defined according to Balogh et al. (1999) as the ratio between the flux density measured on the red side and blue side of the Ca II break, is an indicator of the presence of young stars or of nonstellar emission. Low redshift $(z<0.1)$ red galaxies have $\operatorname{Dn}(4000)=1.98 \pm 0.05$, which is a value that decreases to $=1.95 \pm 0.05$ for $0.1<z<$ 0.15 galaxies (Capetti \& Raiteri 2015).

The concentration index $C_{r}$, which is defined as the ratio of the radii including $90 \%$ and $50 \%$ of the light in the $r$ band, can be used for a morphological classification of galaxies, in which early-type galaxies have higher values of $C_{r}$ than latetype galaxies. Two thresholds have been suggested to define ETGs: a more conservative value at $C_{r} \gtrsim 2.86$ (Nakamura et al. 2003; Shen et al. 2003) and a more relaxed selection at $C_{r} \gtrsim 2.6$ (Strateva et al. 2001; Kauffmann et al. 2003; Bell et al. 2003). Bernardi et al. (2010) found that the second threshold of the concentration index corresponds to a mix of $\mathrm{E}+\mathrm{S} 0+\mathrm{Sa}$ types, while the first mainly selects ellipticals galaxies, removing the majority of Sas, but also some Es and SOs. 

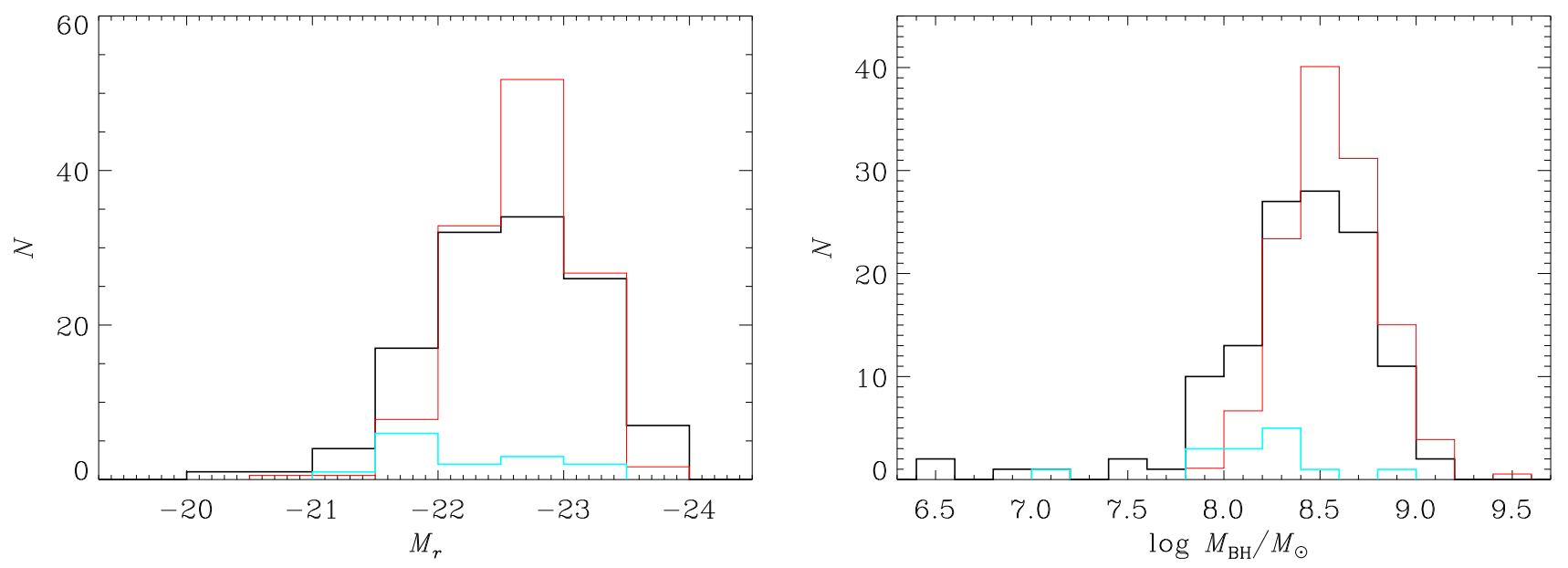

Fig. 1. Distributions of the $r$-band absolute magnitude (left) and black hole masses (right). The black histograms are for FRIICAT (cyan for the HEG FRIICAT subsample), the red histograms for the FRICAT. The FRICAT histograms are all scaled by the relative number of FR I and FR II, i.e., by $122 / 219$.
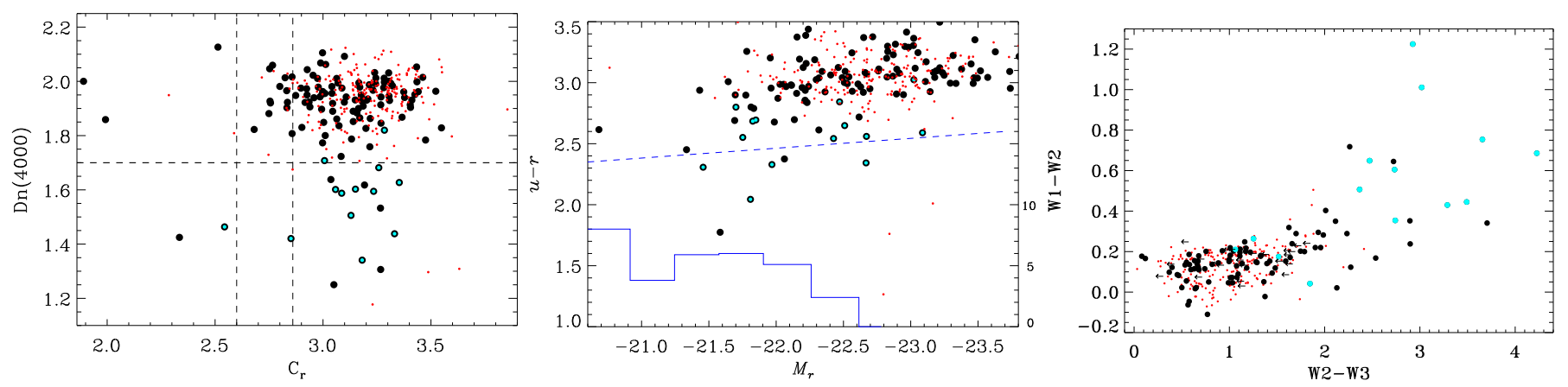

Fig. 2. Left: concentration index $C_{r}$ vs. Dn(4000) index for the FRIICAT and FRICAT samples indicated by black and red dots, respectively. The HEG FR II are represented by cyan circles. Center: absolute $r$-band magnitude, $M_{r}$, vs. $u-r$ color. Right: WISE mid-IR colors.

In Fig. 2 we show the concentration index $C_{r}$ versus the Dn(4000) index (left panel) for the FRIICAT sources. More than $\sim 90 \%$ of the LEG FR II hosts lie in the region of high $C_{r}$ and Dn(4000) values, indicating that they are red ETGs. The HEG FR II are still ETGs, but they show generally lower values of $\operatorname{Dn}(4000)$.

We also consider the $u-r$ color of the galaxies, obtained from SDSS imaging and thus referred to the whole galaxy rather than just the $3^{\prime \prime}$ circular region covered by the SDSS spectroscopic aperture. In Fig. 2 (central panel) we show the $u-r$ color versus the absolute $r$-band magnitude $M_{r}$ of the hosts. As already found by considering the Dn(4000) index, the FR II HEGs show bluer color than the FR II LEGs; in this latter class, only two sources have a $u-r$ color that is smaller than the threshold separating red and blue ETGs (Schawinski et al. 2009).

In Fig. 2, right panel, we show the comparison of the WISE mid-IR colors of FRIICAT and FRICAT sources; the associations with the WISE catalog are computed by adopting a 3".3 search radius (D'Abrusco et al. 2013). All but one of the $(\mathrm{J} 1121+5344)$ FRIICAT sources have a WISE counterpart, but 25 of these sources are undetected in the $W 3$ band. The LEG FR IIs have mid-IR colors similar to those of the FRICAT sources; their mid-IR emission is dominated by their host galaxies since they fall in the same region of elliptical galaxies (Wright et al. 2010). Only five LEGs have $W 2-W 3>2.5$, exceeding the highest value measured for FR Is.

Conversely, HEGs reach mid-IR colors as high as $W 2-W 3=$ 4.3 , colors similar to those of Seyfert and starburst galaxies (e.g., Stern et al. 2005). Their red colors are likely due to a combination of star-forming regions and/or emission from hot dust within a circumnuclear dusty torus.

Overall, we found 10 LEG FR IIs whose properties do not conform with the general behavior of their class, for example, showing blue colors or being associated with small black holes. In some cases, this is due to relatively large errors particularly in the measurement of $\sigma_{*}$, a possible uncertain identification of their spectroscopic class, or a substantial contribution from a bright nonthermal nucleus. However, there are three objects (namely $\mathrm{J} 0755+5204, \mathrm{~J} 1158+3006$, and $\mathrm{J} 1226+2538$ ) for which we obtain estimates of the black hole mass of $\log M_{\mathrm{BH}} \sim$ 6.5-6.8; based on their $C_{r}$ value these objects are late-type galaxies and two of them also show blue optical colors (and red mid-IR colors). These properties are all typical of radio quiet AGN. This contrasts with the observed radio power $\left(\log v L_{r} \sim\right.$ 40.5-40.9) and morphology.

\subsection{Radio properties}

The radio luminosity at $1.4 \mathrm{GHz}$ of the FRIICAT covers the range $L_{1.4}=v_{\mathrm{r}} l_{\mathrm{r}} \sim 10^{39.5}-10^{42.5} \mathrm{erg} \mathrm{s}^{-1}$ (Fig. 3, left panel), reaching a radio power almost two orders of magnitude lower than the FR IIs in the 3C sample. The HEGs are brighter than LEGs (with median of $\log L_{1.4}=41.37$ and 40.76, respectively) and LEGs are brighter than the FRICAT sources by a factor $\sim 3 ; 90 \%$ of the FRIICAT fall below the separation between FR Is and FR IIs originally reported by Fanaroff \& Riley (1974) which translates, with our adopted cosmology and by assuming a spectral index of 0.7 between $178 \mathrm{MHz}$ and $1.4 \mathrm{GHz}$, into $L_{1.4} \sim 10^{41.6} \mathrm{erg} \mathrm{s}^{-1}$. 

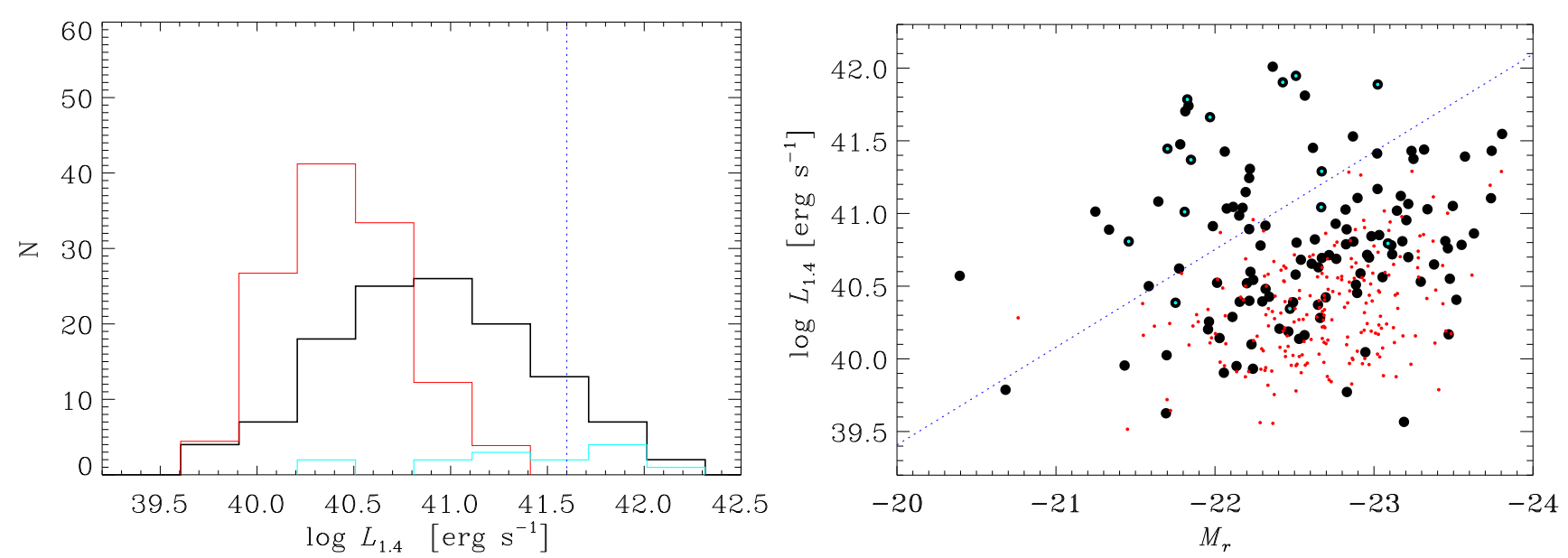

Fig. 3. Left panel: radio luminosity distribution of the FRIICAT (black, cyan for the HEGs) and FRICAT sources (red). The dotted vertical line indicates the transition power between FR I and FR II reported by Fanaroff \& Riley (1974). Right panel: radio luminosity (NVSS) vs. host absolute magnitude, $M_{r}$, for FRIICAT and FRICAT (black and red, respectively; cyan for the HEG FR II). The dotted line shows the separation between FR I and FR II reported by Ledlow \& Owen (1996) to which we applied a correction of 0.34 mag to account for the different magnitude definition and the color transformation between the SDSS and Cousin systems.

Similarly, we find that $\sim 75 \%$ of the FRIICAT sources (and including also four HEGs) are located below the dividing line in the optical-radio luminosity plane defined by Ledlow \& Owen $(1996)^{2}$; see Fig. 3, right panel.

FR IIs show a large spread in both radio and [O III] line luminosities (see Fig. 4). In this plane, the FR II LEGs cover essentially the same region of the FR Is with just a tail toward higher power both in line and in radio; no correlation is seen between these two quantities. The FR II HEGs generally have higher ratios between $L_{[\mathrm{O} \text { III] }}$ and $L_{1.4}$ than LEGs, which is an effect already found in the 3C sample (Buttiglione et al. 2010). The HEGs in FRIICAT are mostly located above the correlation defined by the 3C HEGs. A linear fit including both samples is indeed shallower (with a slope of 0.91) than that obtained from the $3 \mathrm{C}$ sources alone (whose slope is 1.15).

\subsection{Comparison with previous works}

As discussed in Sect. 2, we decided to maintain the traditional morphological visual classification into edge-brightened FR IIs and edge-darkened FR Is rather than adopting quantitative methods such as those used by Lin et al. (2010). The comparison of our classification with that proposed by these authors indicates that, among the 96 sources in common, $\sim 80 \%$ of the FRIICAT sources are classified as class $a$ in their nomenclature, i.e., sources with two hot spots on either side of the galaxy. Most of the remaining objects fall in class $b$, in which the emission peak is coincident with the host galaxy; however, the inspection of these FR II radio sources does not show any clear distinguishing feature from those in the main class, other than having a relatively brighter central source in addition to the two lobes.

The main drawback of our scheme, based on the subjective visual inspection of radio images, is the relatively high fraction of sources of uncertain classification. The rather strict criteria adopted for a positive classification as FR I in Capetti et al. (2017) and here for the FR II enabled us to select only 219 FR Is and 122 FR II; more than half of the 714 radio galaxies extended more than $30 \mathrm{kpc}$ cannot be allocated to any FR class. On the

\footnotetext{
2 We shifted the dividing line to the right of the diagram to include a correction of $0.12 \mathrm{mag}$ to scale our total host magnitude to the $\mathrm{M}_{24.5}$ used by these authors, and an additional 0.22 mag to convert the Cousin system into the SDSS system (Fukugita et al. 1996).
}

other hand, this strategy allows us to select samples that are very uniform from a morphological point of view and that are optimally suited for our main purpose, i.e., the comparison of the properties of the two classes.

More recently, Miraghaei \& Best (2017) performed an analysis on the same initial sample, with an apparently similar selection strategy based on visual inspection. However, the resulting sample of both FR I and FR II differ significantly from those we obtained with only $\sim 25 \%$ of objects in common for both classes, even restricting the comparison to the same range of redshift, $0.03<z<0.15$. This mismatch is likely due to the different requirements (based, e.g., on linear instead of angular sizes and different radio flux limits); most importantly they considered only sources corresponding to multicomponents objects in FIRST and this rejects most of the edge darkened sources we included in FRICAT. Overall, their results do not strongly differ from ours, probably because (leaving aside the HEGs) the properties of low $z$ radio AGN are very homogeneous regardless of their radio morphology. However, for example, we do not find significative differences in the $C_{r}$ values between FRI and FRII hosts.

\section{Discussion and conclusions}

The properties of the FRIICAT sources differ between those spectroscopically identified as LEGs or HEGs. The HEGs have lower optical luminosities, smaller black hole masses, and higher radio luminosities with respect to LEGs, although a substantial overlap between the two classes exist for all these quantities. The clearest differences are related to the ratio between line and radio luminosities and to their colors; HEGs are bluer in the optical and redder in the mid-IR. These results confirm the conclusions of previous studies (e.g., Baldi \& Capetti 2008; Buttiglione et al. 2010; Baldi \& Capetti 2010; Best \& Heckman 2012).

The population of the LEG FR IIs included in the FRIICAT is remarkably uniform. They are all luminous red ETGs with large black hole masses $\left(M_{\mathrm{BH}} \gtrsim 10^{8} M_{\odot}\right)$; only $\sim 10 \%$ of the LEG FR IIs depart from this general description. All these properties are shared with the hosts of the FRICAT sources. The distributions of $M_{\mathrm{BH}}$ and $M_{r}$ differ with a statistical significance higher than $95 \%$ according to the Kolmogoroff-Smirnov test; this is due to the presence of a tail of low $M_{\mathrm{BH}}$ sources, reaching value as 


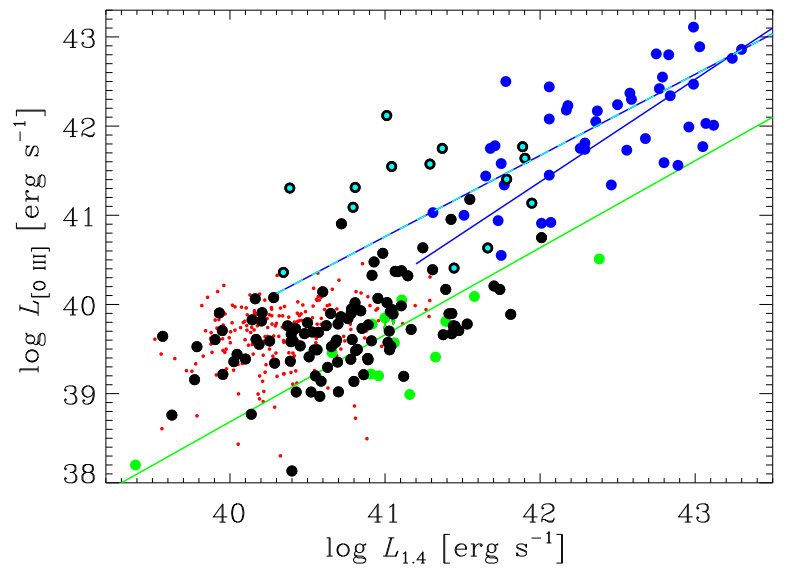

Fig. 4. Radio (NVSS) vs. [O III] line luminosity of the FRIICAT (black), FRICAT (red), 3C-FR I (green), and 3C-FR II samples (blue) The green line (blue). shows the linear correlation between these two quantities derived from the FR Is (FR IIs) of the 3C sample from (Buttiglione et al. 2010). The dashed blue-cyan line is instead the linear fit on both the 3C and the FRIICAT HEGs.

low as $3 \times 10^{6} M_{\odot}$. However, the median of $M_{\mathrm{BH}}$ and $M_{r}$ differ only marginally by less than 0.1 dex. Even the median radio luminosity of LEG FR II is just a factor $\sim 3$ higher than that measured in FR I. Apparently, the difference in radio morphology between edge-brightened and edge-darkened radio sources does not translate into a clear separation between the nuclear and host properties, while the spectroscopic classes, LEG and HEG, do.

The FRIICAT sample unveils a population of FR IIs of much lower radio power with respect to those obtained at high radio flux thresholds, extending it downward by two orders of magnitude. The correspondence of the morphological classification of FR I and FR II with a separation in radio power that is observed, for example, in the 3C sample, disappears. This conclusion is in line with previous results (Best 2009; Lin et al. 2010; Wing \& Blanton 2011). A radio source produced by a low power jet can be edge brightened or edge darkened and the outcome is not due to differences in the optical properties of the host galaxy.

Nonetheless, Capetti et al. (2017) find that the connection between radio morphology and host properties is preserved in FR Is; there is a well-defined threshold of radio power above which an edge darkened radio source does not form and this limit has a strong positive dependence on the host luminosity. This effect was originally seen by Ledlow \& Owen (1996) but partly lost in subsequent studies; we believe that we recover it because of the stricter criteria we adopted for the selection of FR Is.

It can be envisaged that brighter galaxies are associated with denser and more extended hot coronae that are able to disrupt more powerful jets. But the large population of low power FR IIs indicates that the situation is more complex; there is a large overlap of radio power between FR Is and FR IIs and radio power is believed to be a robust proxy for the jet power (e.g., Willott et al. 1999; Bîrzan et al. 2004). Apparently, jets of the same power expanding in similar galaxies can form both FR I and FR II. This indicates that the optical properties of the host and radio luminosity are not the only parameters driving the evolution of low power radio sources. Further studies of, for example, the X-rays properties and the larger scale environment are needed to clarify this issue.

Acknowledgements. F.M. gratefully acknowledges the financial support of the Programma Giovani Ricercatori - Rita Levi Montalcini - Rientro dei Cervelli (2012) awarded by the Italian Ministry of Education, Universities and Research (MIUR). Part of this work is based on the NVSS (NRAO VLA Sky Survey): The
National Radio Astronomy Observatory is operated by Associated Universities, Inc., under contract with the National Science Foundation. This publication makes use of data products from the Wide-field Infrared Survey Explorer, which is a joint project of the University of California, Los Angeles, and the Jet Propulsion Laboratory/California Institute of Technology, funded by the National Aeronautics and Space Administration. This research made use of the NASA/IPAC Infrared Science Archive and Extragalactic Database (NED), which are operated by the Jet Propulsion Laboratory, California Institute of Technology, under contract with the National Aeronautics and Space Administration. We acknowledge the usage of the HyperLeda database (http: //leda.univ-lyon1.fr). Funding for SDSS-III has been provided by the Alfred P. Sloan Foundation, the Participating Institutions, the National Science Foundation, and the US Department of Energy Office of Science. The SDSSIII website is http://www.sdss3.org/. SDSS-III is managed by the Astrophysical Research Consortium for the Participating Institutions of the SDSS-III Collaboration, including the University of Arizona, the Brazilian Participation Group, Brookhaven National Laboratory, University of Cambridge, Carnegie Mellon University, University of Florida, the French Participation Group, the German Participation Group, Harvard University, the Instituto de Astrofisica de Canarias, the Michigan State/Notre Dame/JINA Participation Group, Johns Hopkins University, Lawrence Berkeley National Laboratory, Max Planck Institute for Astrophysics, Max Planck Institute for Extraterrestrial Physics, New Mexico State University, New York University, Ohio State University, Pennsylvania State University, University of Portsmouth, Princeton University, the Spanish Participation Group, University of Tokyo, University of Utah, Vanderbilt University, University of Virginia, University of Washington, and Yale University.

\section{References}

Abazajian, K. N., Adelman-McCarthy, J. K., Agüeros, M. A., et al. 2009, ApJS, 182,543

Baldi, R. D., \& Capetti, A. 2008, A\&A, 489, 989

Baldi, R. D., \& Capetti, A. 2010, A\&A, 519, A48

Balogh, M. L., Morris, S. L., Yee, H. K. C., Carlberg, R. G., \& Ellingson, E. 1999, ApJ, 527, 54

Becker, R. H., White, R. L., \& Helfand, D. J. 1995, ApJ, 450, 559

Bell, E. F., McIntosh, D. H., Katz, N., \& Weinberg, M. D. 2003, ApJS, 149, 289 Bernardi, M., Shankar, F., Hyde, J. B., et al. 2010, MNRAS, 404, 2087

Best, P. N. 2009, Astron. Nachr., 330, 184

Best, P. N., \& Heckman, T. M. 2012, MNRAS, 421, 1569

Bîrzan, L., Rafferty, D. A., McNamara, B. R., Wise, M. W., \& Nulsen, P. E. J. 2004, ApJ, 607, 800

Brinchmann, J., Charlot, S., White, S. D. M., et al. 2004, MNRAS, 351, 1151

Buttiglione, S., Capetti, A., Celotti, A., et al. 2010, A\&A, 509, A6

Capetti, A., \& Raiteri, C. M. 2015, A\&A, 580, A73

Capetti, A., Massaro, F., \& Baldi, R. D. 2017, A\&A, 598, A49

Condon, J. J., Cotton, W. D., Greisen, E. W., et al. 1998, AJ, 115, 1693

D'Abrusco, R., Massaro, F., Paggi, A., et al. 2013, ApJS, 206, 12

D'Abrusco, R., Massaro, F., Paggi, A., et al. 2014, ApJS, 215, 14

Fanaroff, B. L., \& Riley, J. M. 1974, MNRAS, 167, 31

Fukugita, M., Ichikawa, T., Gunn, J. E., et al. 1996, AJ, 111, 1748

Hardcastle, M. J., Evans, D. A., \& Croston, J. H. 2007, MNRAS, 376, 1849

Helfand, D. J., White, R. L., \& Becker, R. H. 2015, ApJ, 801, 26

Kauffmann, G., Heckman, T. M., White, S. D. M., et al. 2003, MNRAS, 341, 33

Kewley, L. J., Groves, B., Kauffmann, G., \& Heckman, T. 2006, MNRAS, 372, 961

Laing, R. A., Jenkins, C. R., Wall, J. V., \& Unger, S. W. 1994, in The Physics of Active Galaxies, eds. G. V. Bicknell, M. A. Dopita, \& P. J. Quinn, ASP Conf. Ser., 54, 201

Ledlow, M. J., \& Owen, F. N. 1996, AJ, 112, 9

Lin, Y.-T., Shen, Y., Strauss, M. A., Richards, G. T., \& Lunnan, R. 2010, ApJ, 723,1119

Miraghaei, H., \& Best, P. N. 2017, MNRAS

Nakamura, O., Fukugita, M., Yasuda, N., et al. 2003, AJ, 125, 1682

Owen, F. N., \& Rudnick, L. 1976, ApJ, 205, L1

Planck Collaboration XIII. 2016, A\&A, 594, A13

Schawinski, K., Lintott, C., Thomas, D., et al. 2009, MNRAS, 396, 818

Shen, S., Mo, H. J., White, S. D. M., et al. 2003, MNRAS, 343, 978

Smolčić, V. 2009, ApJ, 699, L43

Stern, D., Eisenhardt, P., Gorjian, V., et al. 2005, ApJ, 631, 163

Strateva, I., Ivezić, Ž., Knapp, G. R., et al. 2001, AJ, 122, 1861

Tremaine, S., Gebhardt, K., Bender, R., et al. 2002, ApJ, 574, 740

Tremonti, C. A., Heckman, T. M., Kauffmann, G., et al. 2004, ApJ, 613, 898

Willott, C. J., Rawlings, S., Blundell, K. M., \& Lacy, M. 1999, MNRAS, 309, 1017

Wing, J. D., \& Blanton, E. L. 2011, AJ, 141, 88

Wright, E. L., Eisenhardt, P. R. M., Mainzer, A. K., et al. 2010, AJ, 140, 1868 


\section{Appendix A: FIRST images of the FRIICAT sources}
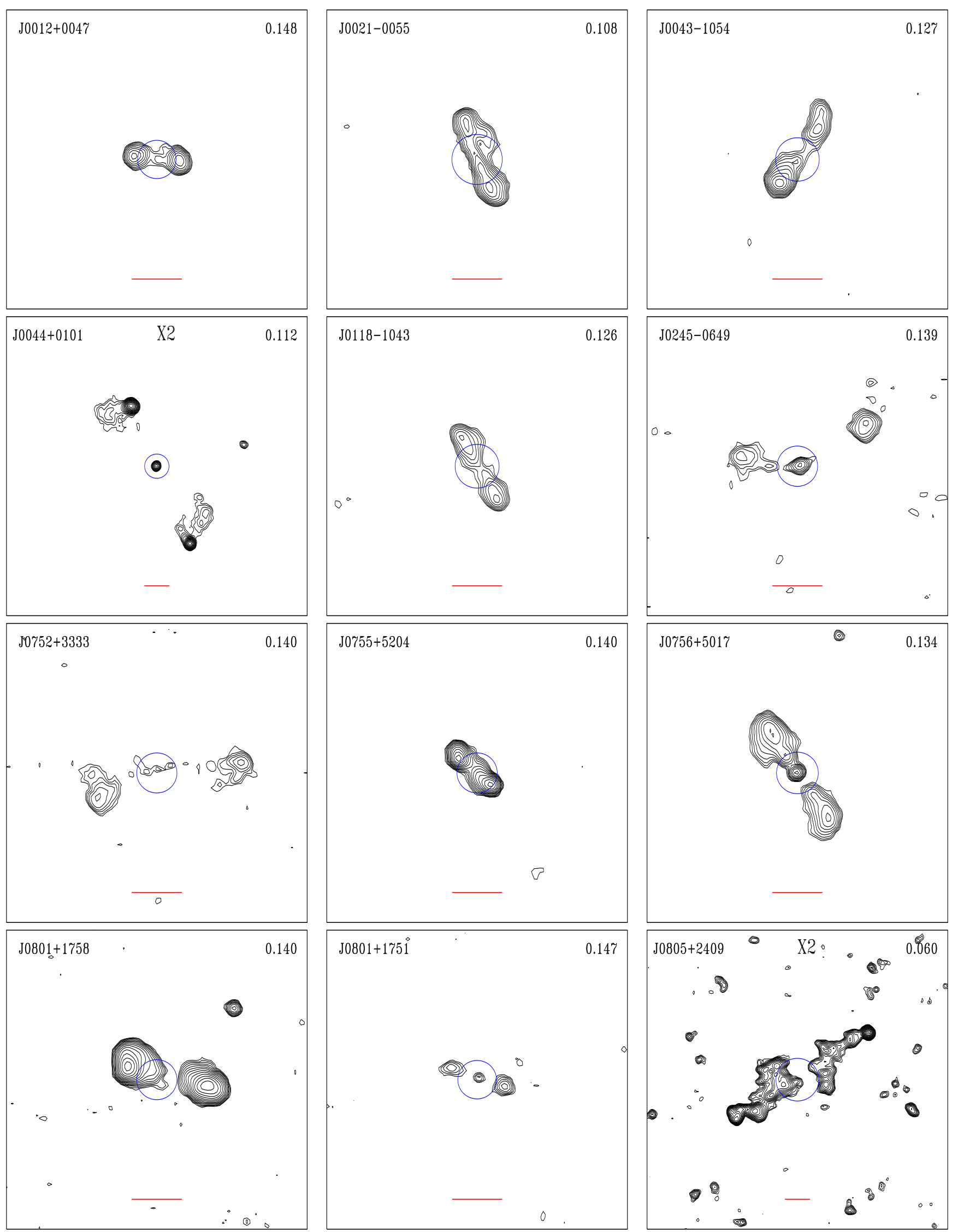

Fig. A.1. Images of the FR IIs selected. Contours are drawn starting from $0.45 \mathrm{mJy} / \mathrm{beam}$ and increase with a geometric progression with a common ratio of $\sqrt{2}$. The field of view is $3^{\prime} \times 3^{\prime}$ in most cases, except for those marked with a ' $\mathrm{X} 2$ ' at the top; the red tick at the bottom is $30^{\prime \prime}$ long. The blue circle is centered on the host galaxy and has a radius of $30 \mathrm{kpc}$. The source ID and redshift are reported in the upper corners. 
A. Capetti et al.: FRIICAT: A FIRST catalog of FR II radio galaxies
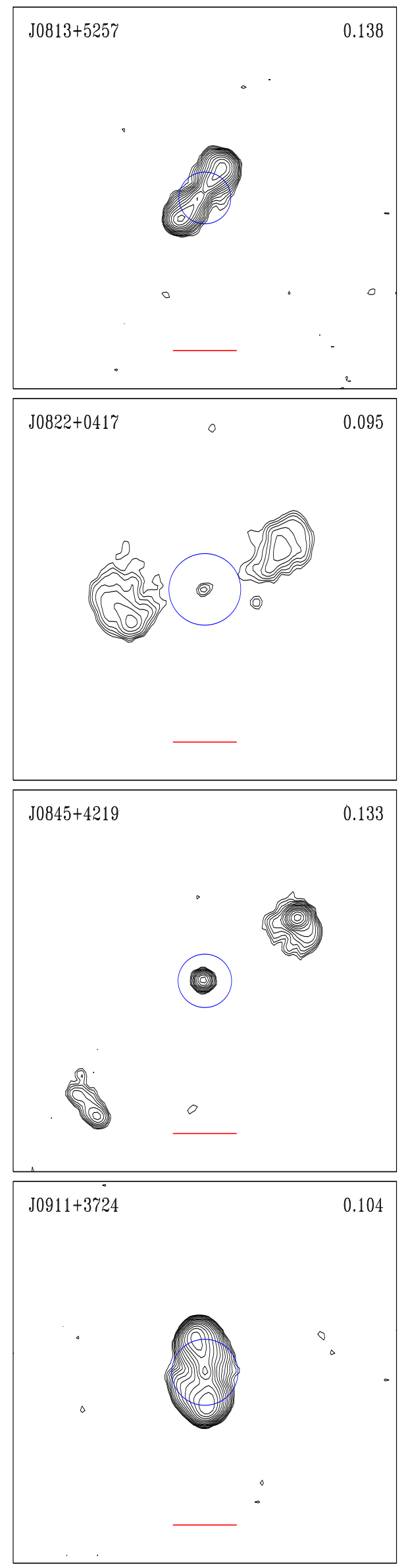

Fig. A.1. continued.

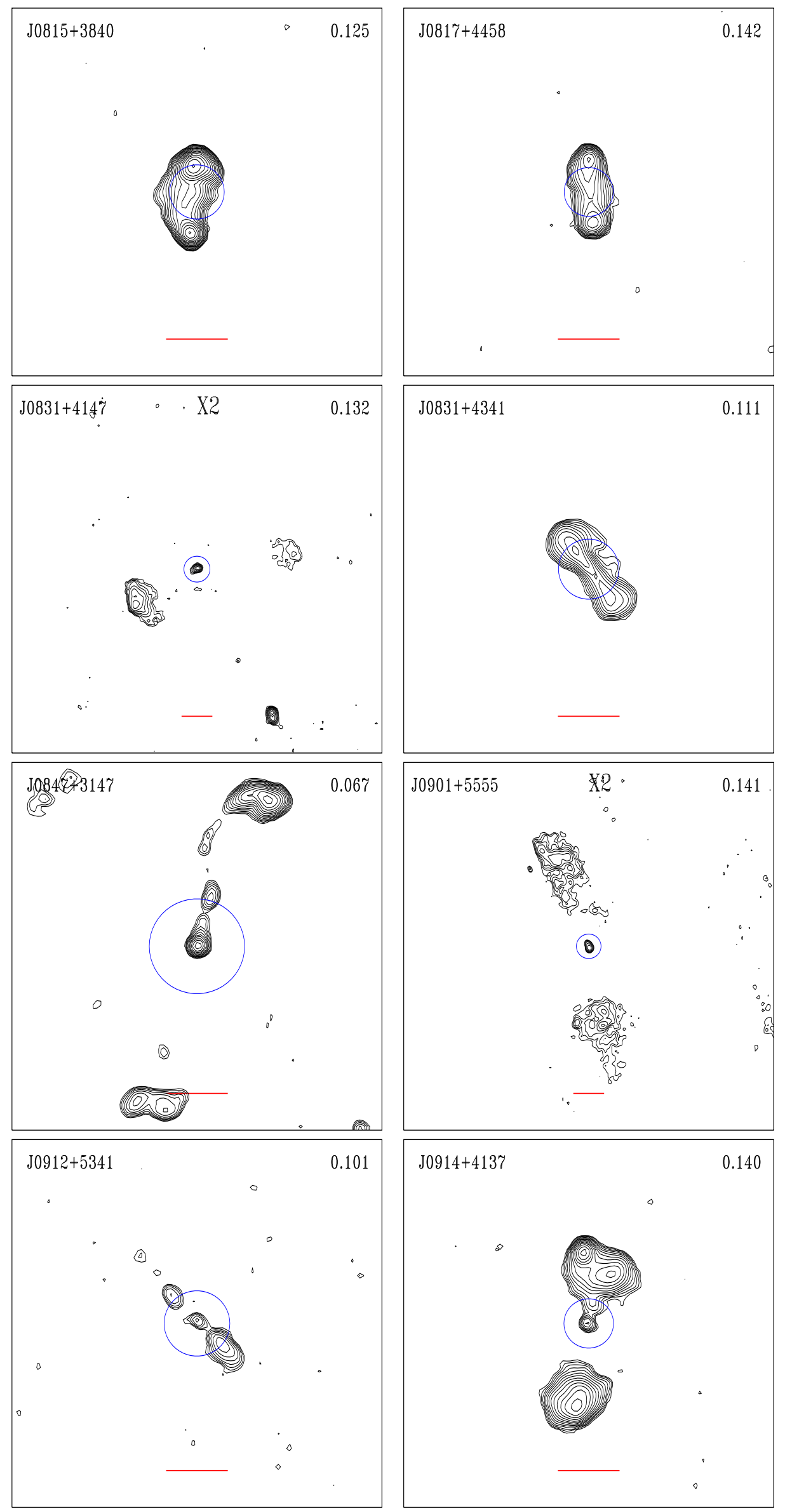

A81, page 7 of 16 
A\&A 601, A81 (2017)

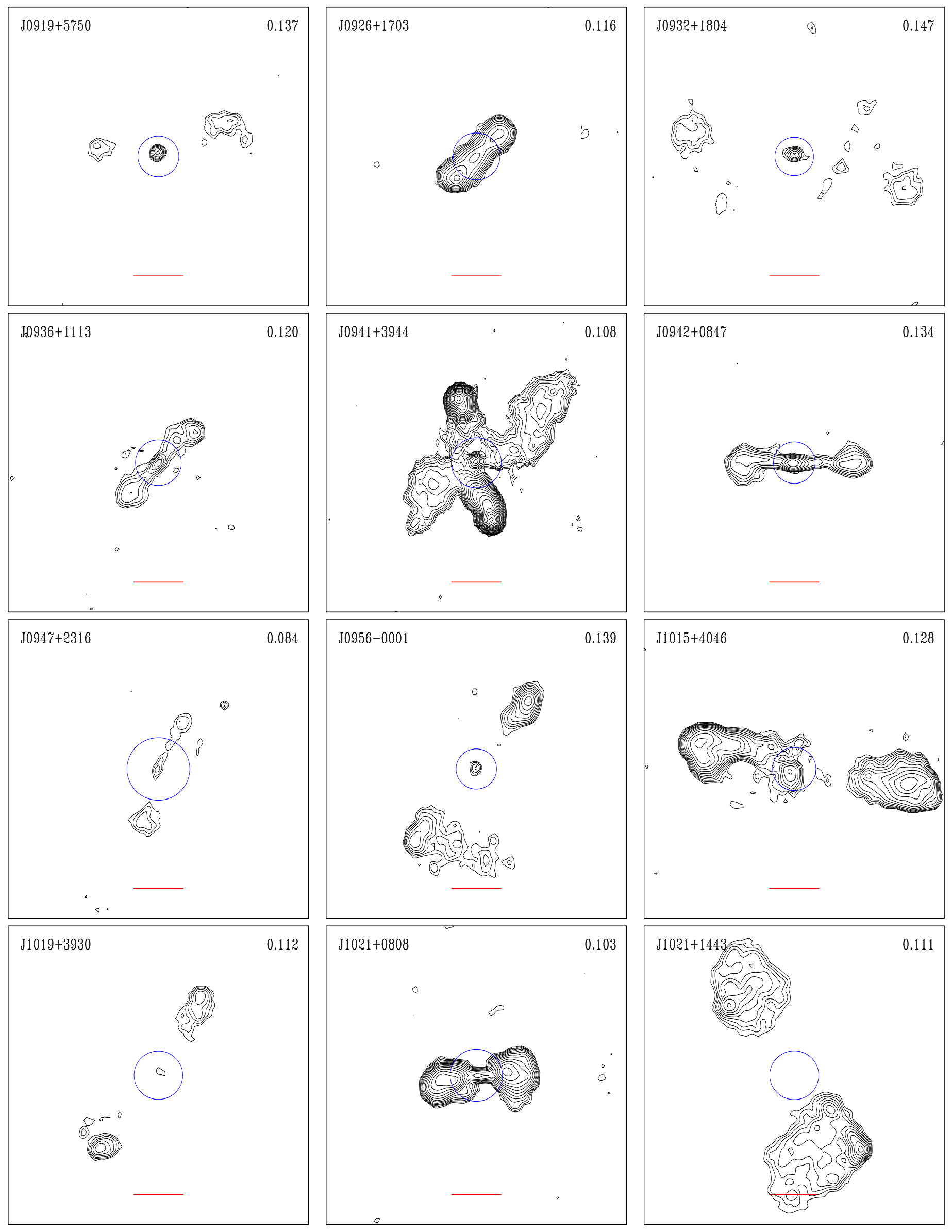

Fig. A.1. continued. 
A. Capetti et al.: FRIICAT: A FIRST catalog of FR II radio galaxies
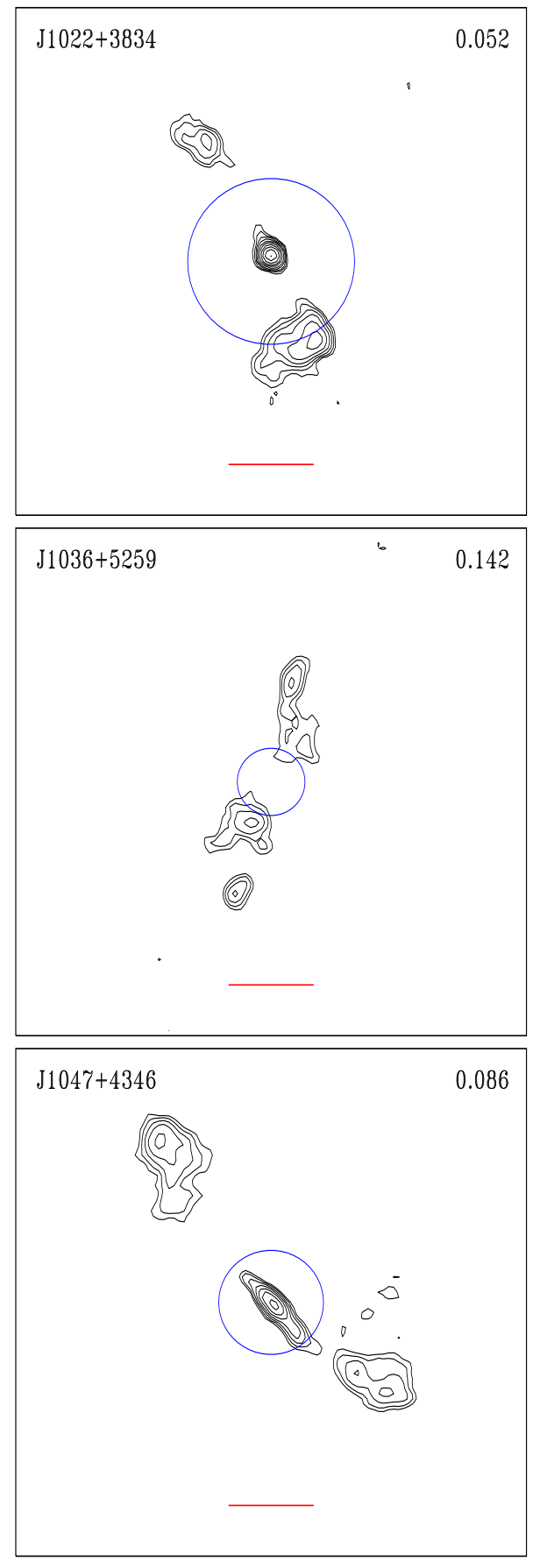

$J 1100+2539$

0.145

0
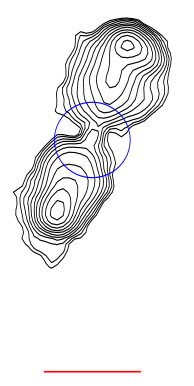
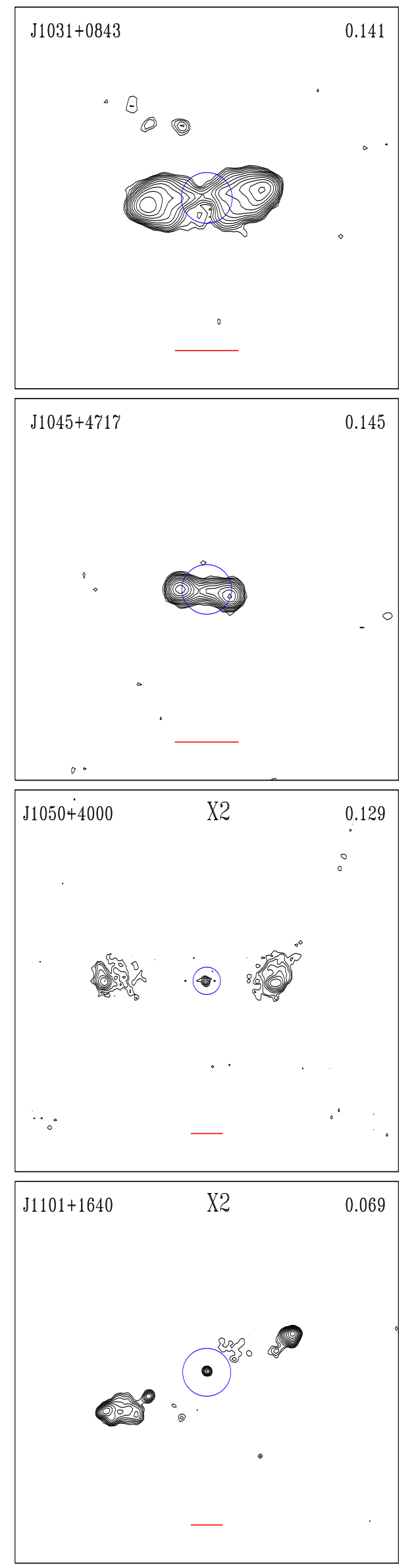

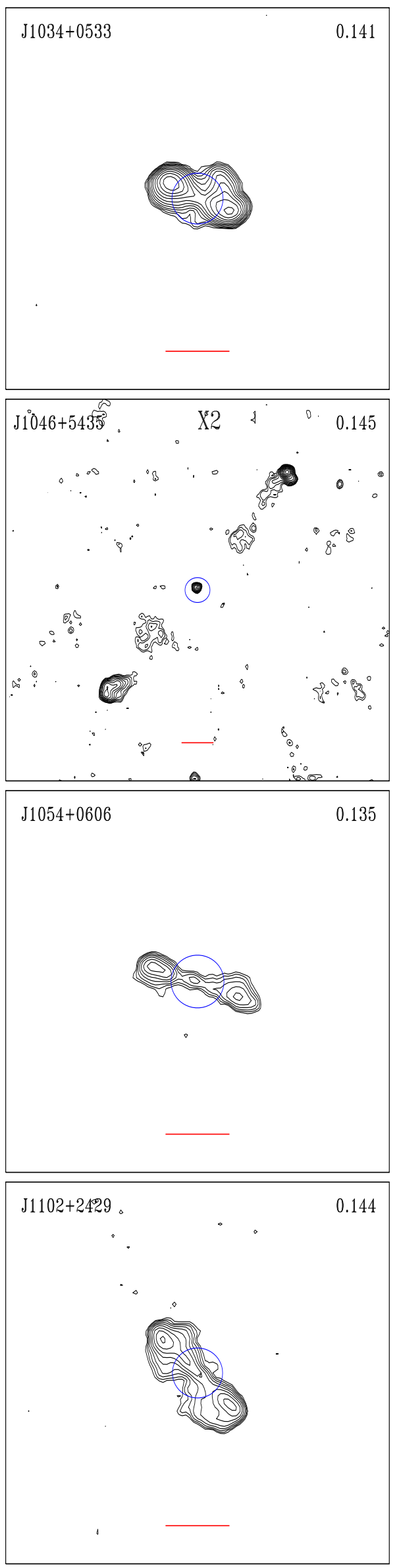

Fig. A.1. continued. 
A\&A 601, A81 (2017)

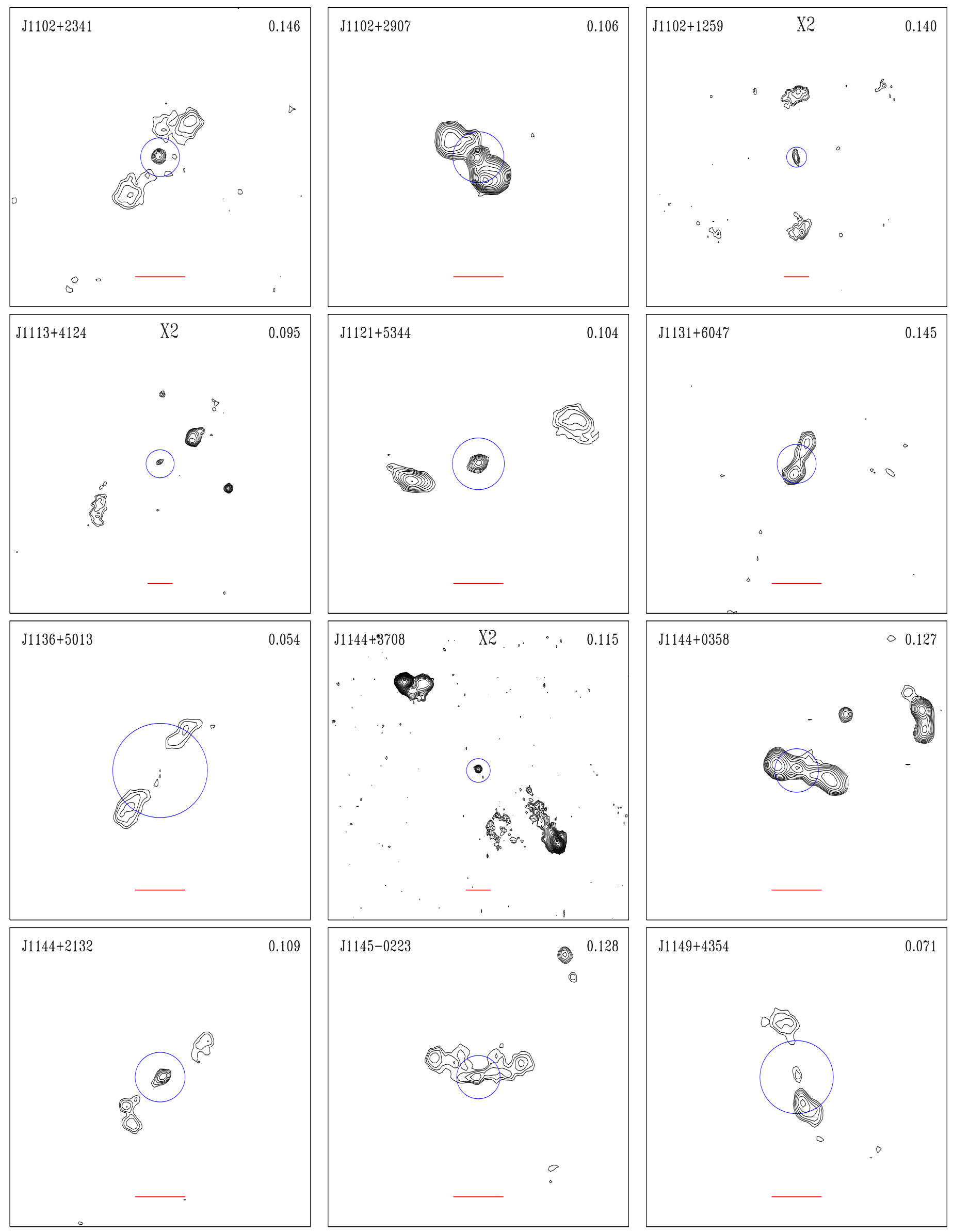

Fig. A.1. continued. 
A. Capetti et al.: FRIICAT: A FIRST catalog of FR II radio galaxies
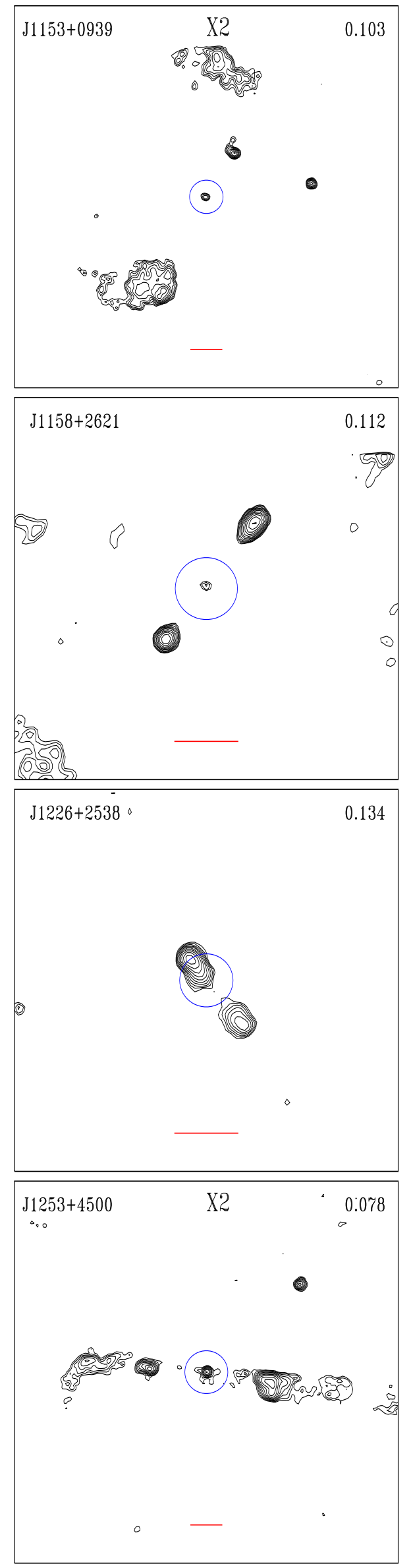

Fig. A.1. continued.

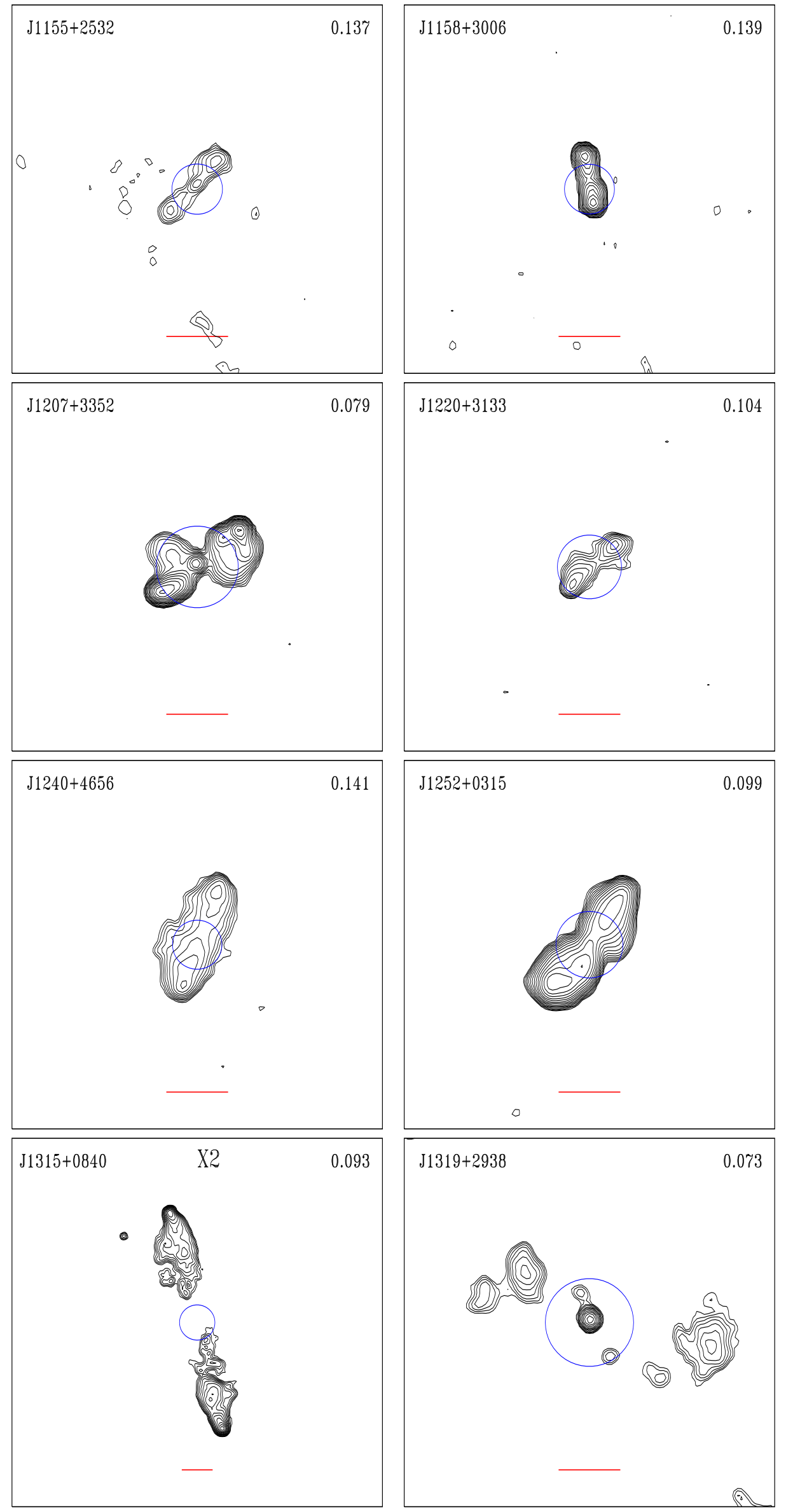

A81, page 11 of 16 
A\&A 601, A81 (2017)
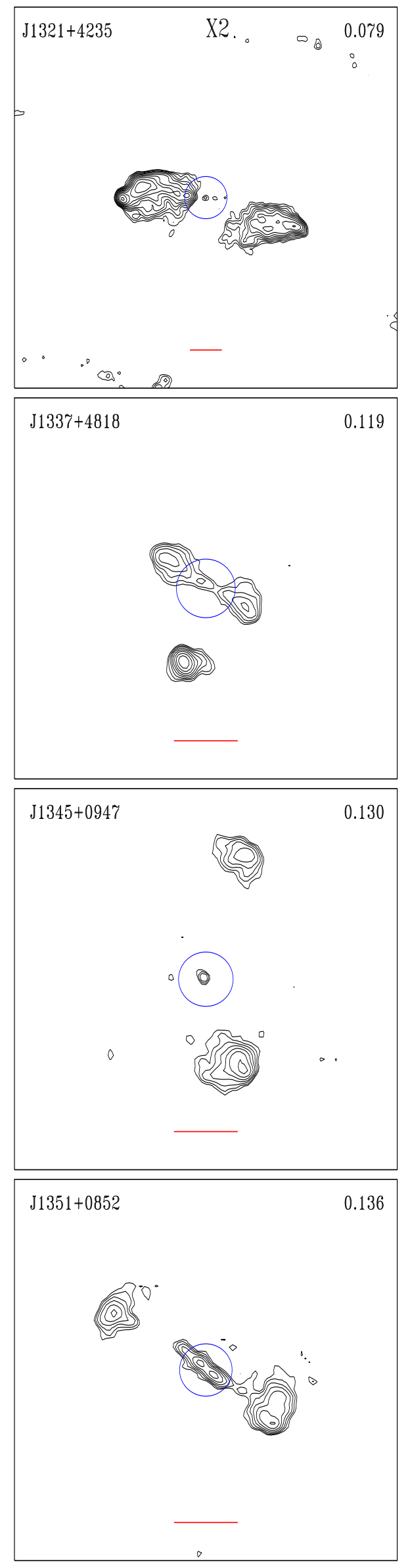

Fig. A.1. continued.

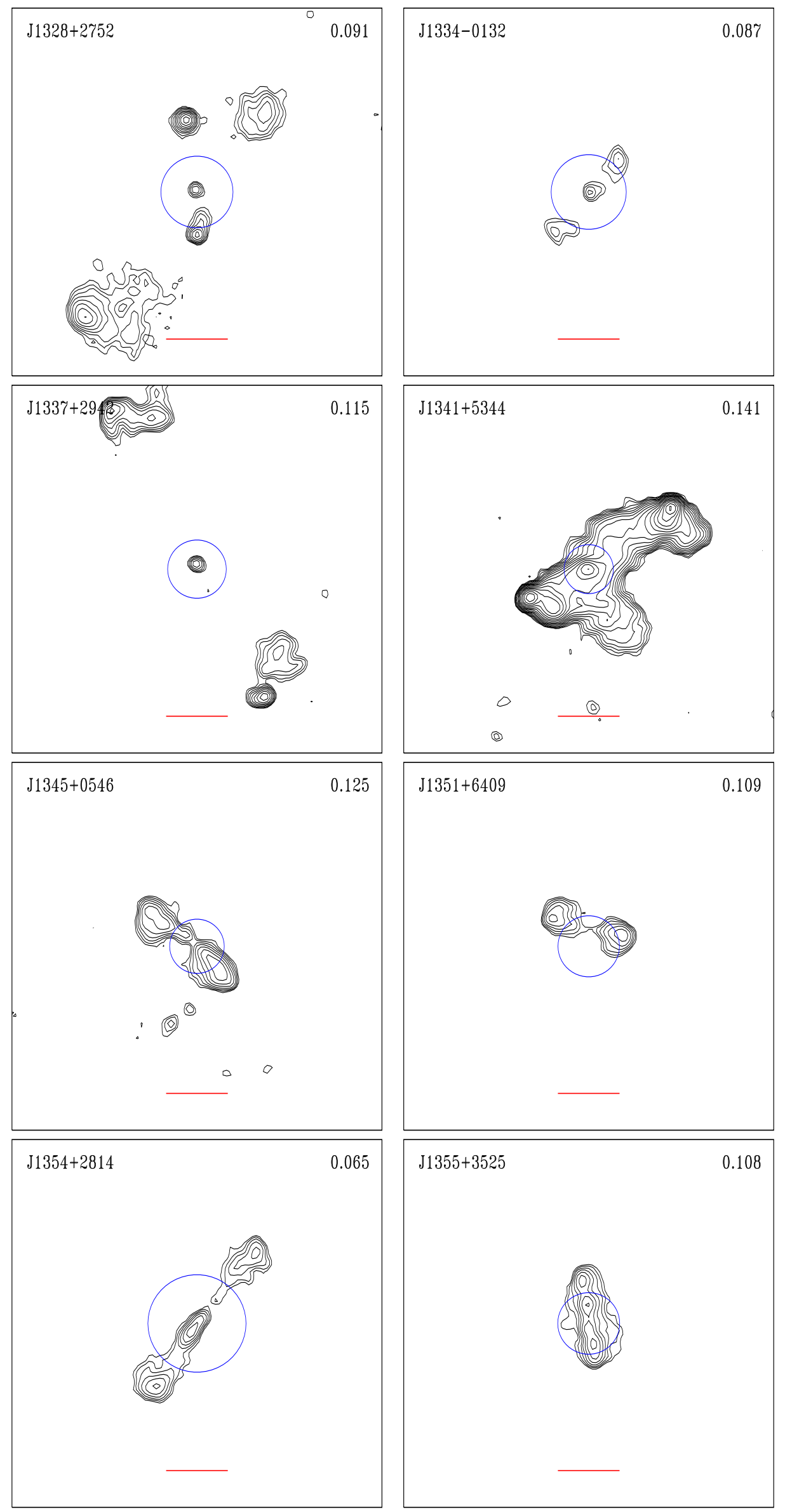


A. Capetti et al.: FRIICAT: A FIRST catalog of FR II radio galaxies
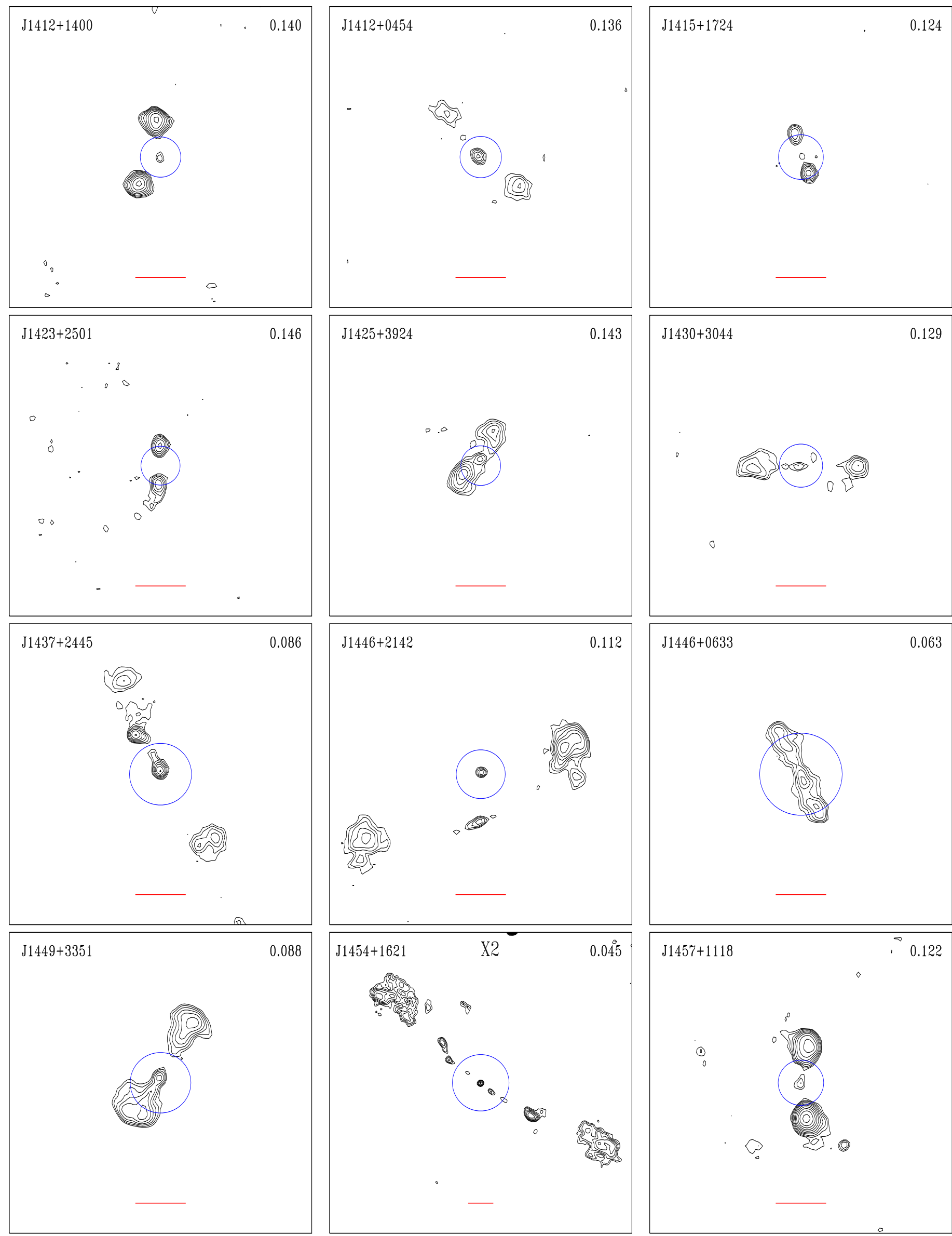

$\mathrm{J} 1430+3044$

0.129

J1449+3351

0.088
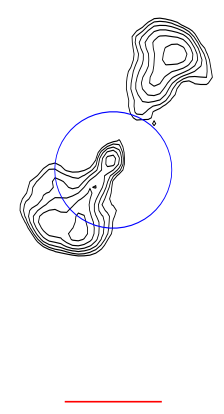

Fig. A.1. continued. 
A\&A 601, A81 (2017)
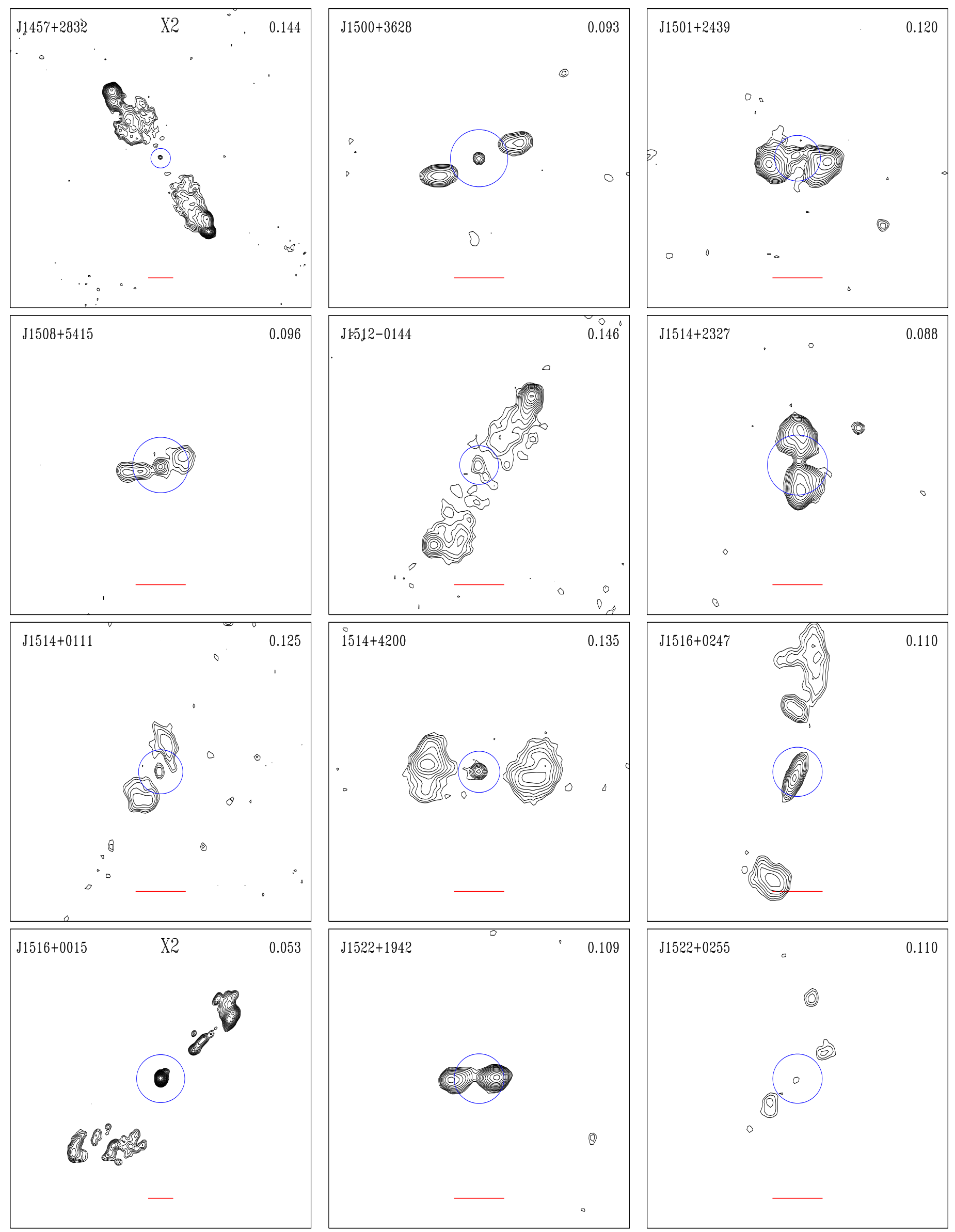

Fig. A.1. continued. 
A. Capetti et al.: FRIICAT: A FIRST catalog of FR II radio galaxies
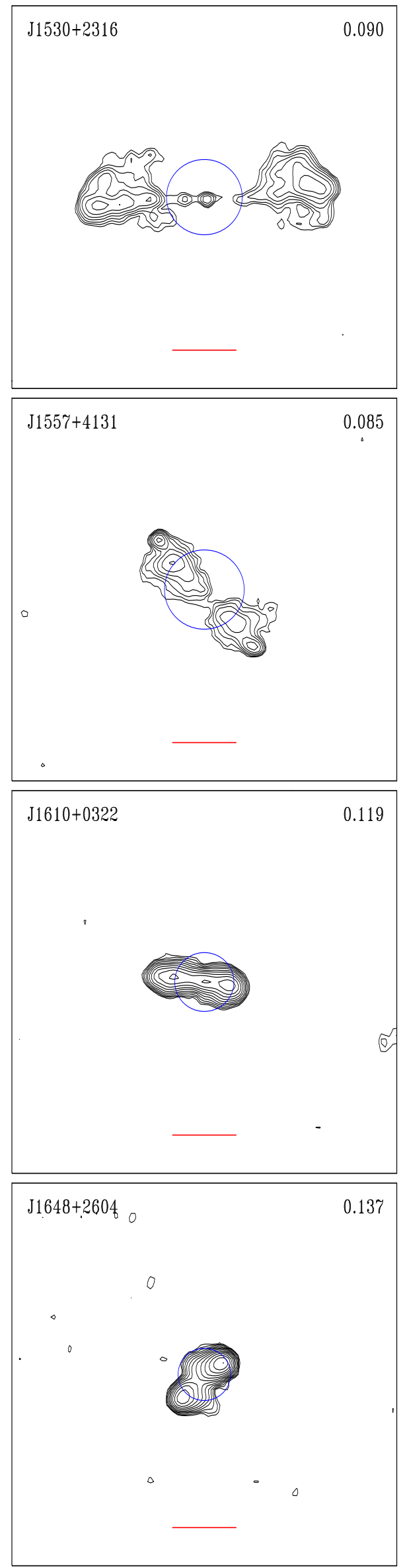

Fig. A.1. continued.

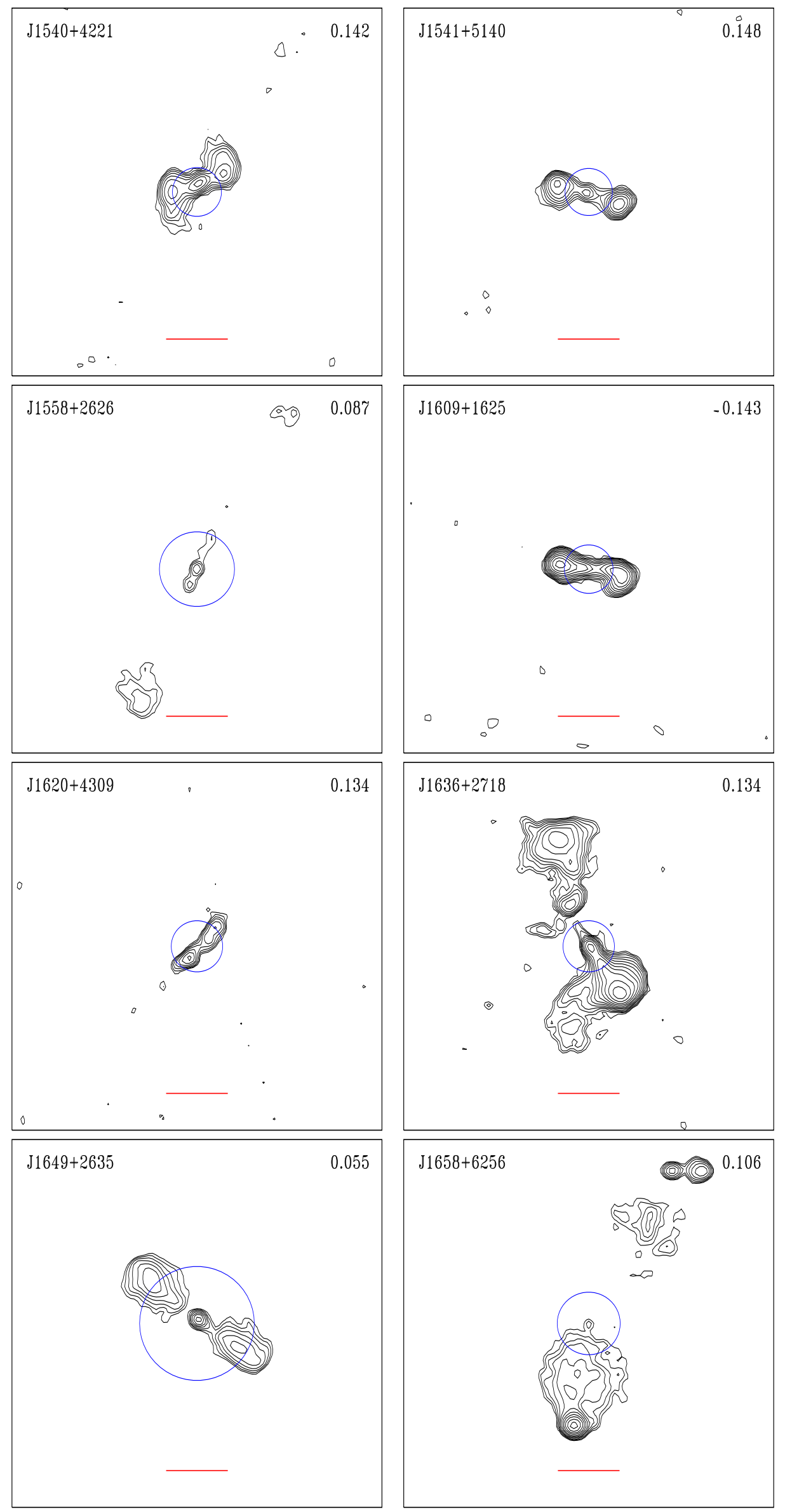

A81, page 15 of 16 
A\&A 601, A81 (2017)
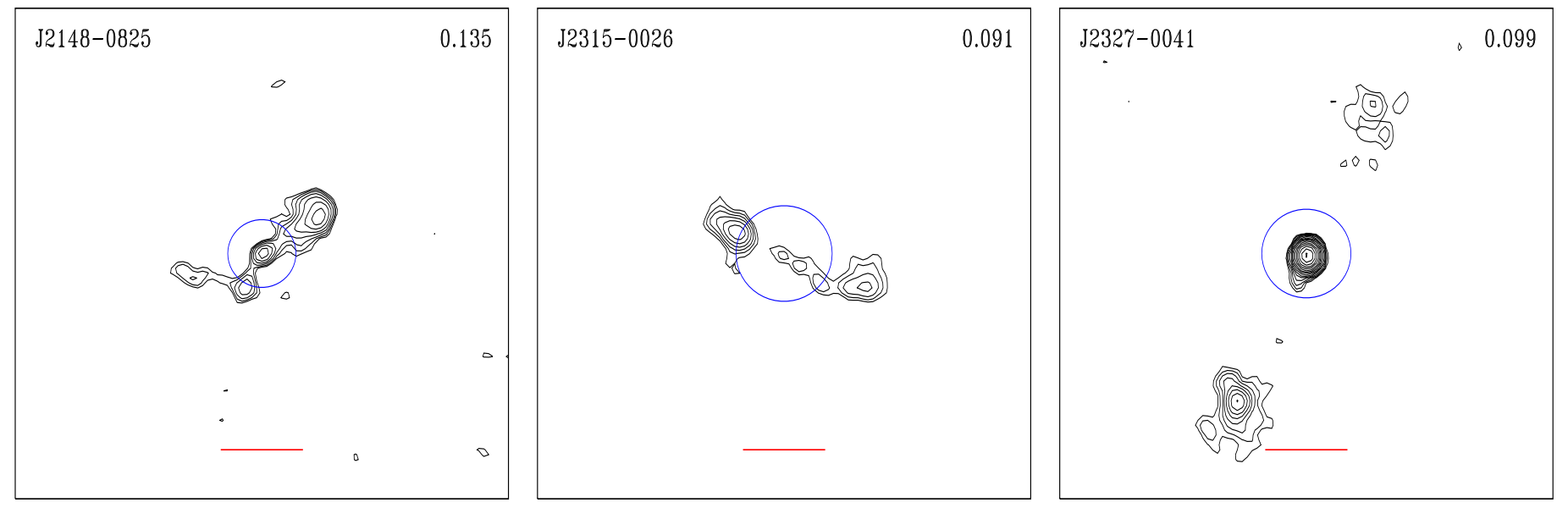

Fig. A.1. continued. 